\title{
The tale of the great cuckoo egg
}

The idea that viruses might cause tumours inadvertently led to the discovery of cellular growth genes that can promote cancer formation - but usually in the absence of viruses. This success rapidly outgrew the original idea.

\section{George Klein}

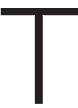
he identification of specific microorganisms as causative agents of infectious diseases generated much optimism around the turn of the last century. It raised hope that diseases of unknown origin, including cancer, could soon be explained in a similar way. When Peyton Rous showed in 1911 that fowl sarcomas could be transmitted with cell-free filtrates, it was believed that the problem was solved: cancer was a viral disease. But, when similar experiments with mouse and rat tumours failed soon after, it was concluded that tumour viruses occurred only in birds, and the field fell into disrepute.

For the next four decades this opinion prevailed. Potentially important positive findings were 'interpreted away'. The discovery in the 1920s of the Shope papilloma virus, which causes warts in rabbits, caused little enthusiasm because the tumours were largely benign. The mouse mammary tumour virus (MMTV), discovered in the 1930s, was called 'milk factor', rather than 'milk virus', to avoid a negative reaction from those grant-giving bodies included - who had relegated tumour viruses to the cabinet of freaks. Those who understood the viral nature of the 'factor' remained unenthused because analysis showed that MMTV was neither necessary nor sufficient for tumour induction. It merely increased the probability of breast cancer in hosts with both a susceptible genetic background and the appropriate hormonal environment.

The great change in the climate of opinion came in the 1950s when Ludvik Gross discovered the mouse leukaemia virus, and Sarah Stewart and Bernice Eddy identified the polyoma virus. Within a few years the pendulum had swung to the opposite extreme. After decades of failed attempts, viruses that could induce tumours in mammals were now isolated in quick succession. Tumour virology rapidly became a 'most favoured nation' with the grantgiving bodies.

The oncogene concept - that tumour viruses carry 'cancer genes' that can transform some of their target cells into a cancerous or pre-cancerous state - was formulated in the context of this enthusiasm. Ironically, the concept was based on the slow acting or chronic (class II) RNA tumour
By discovering cellular genes that

regulate growth and that can

contribute to cancer development

after illegitimate viral activation,

the virologists hatched a great

cuckoo egg. These genes, when

mutated, can promote cancer

formation independent of viruses.

Their discovery relegated tumour

virology once again to a less

prominent place and reaffirmed

the sovereignty of cell biology.

virus which, it turned out later, is the only tumour-virus family that does not carry transforming genes. It is the other two families, the directly acting or acute (class I) RNA tumour viruses and the DNA tumour viruses, that carry oncogenes that can transform cells to a cancerous state.

The class I RNA tumour viruses do not cause tumours in nature, nor can they propagate without the intervention of the scientist. They can induce tumours because they have accidentally incorporated from cells genes that regulate growth. Basically, they are highly informative laboratory artefacts. As their name suggests, they carry their genetic information in RNA. After entering a new host cell, the viral enzyme reverse transcriptase copies the viral RNA into DNA (called provirus DNA) which integrates randomly into the host cell's DNA. When the virus starts to reproduce itself, and the proviral DNA is transcribed back into RNA again, some of the new virus particles may also carry additional, cellular sequences from regions adjacent to the virus's random integration site.

Tumour virologists isolated some 20 previously unknown growth regulatory genes from about 40 viral isolates that favour tumour development. These genes originated from corresponding cellular genes which could themselves contribute to spontaneous tumour development, without any viral intervention, after structural or regulatory mutations.

The other type of RNA virus - class II RNA tumour viruses - do not themselves contain oncogenes, but contribute to malignant tumour development relatively infrequently when their proviral DNA happens to integrate in the host DNA near such cellulargenes.

By discovering cellular genes that regulate growth and that can contribute to cancer development after illegitimate viral activation, the virologists hatched a great cuckoo egg. These genes, when mutated, can promote cancer formation independent of viruses. Their discovery relegated tumour virology once again to a less prominent place and reaffirmed the sovereignty of cell biology. Cancer is a disease of the cellular DNA.

All oncogenes turned out to be highly conserved household genes that participate in the regulation of the cell cycle. Their potentially tumorigenic forms drive the cell towards division. For overt tumour development, additional genetic changes are required, however. These include the loss of cell-cycle checkpoint controls, inhibition of programmed cell death (apoptosis), and upregulation of blood supply. The oncogene field emerged from erroneous concepts combined with good experimentation. The great cuckoo egg has become a rich source of many unexpected discoveries.

George Klein is at the Microbiology and

Tumorbiology Center, Karolinska Institute, PO Box 280, S-171 77 Stockholm, Sweden.

The SV40 virus does not seem to cause tumours in monkeys, its original hosts, but does when injected into hamsters.

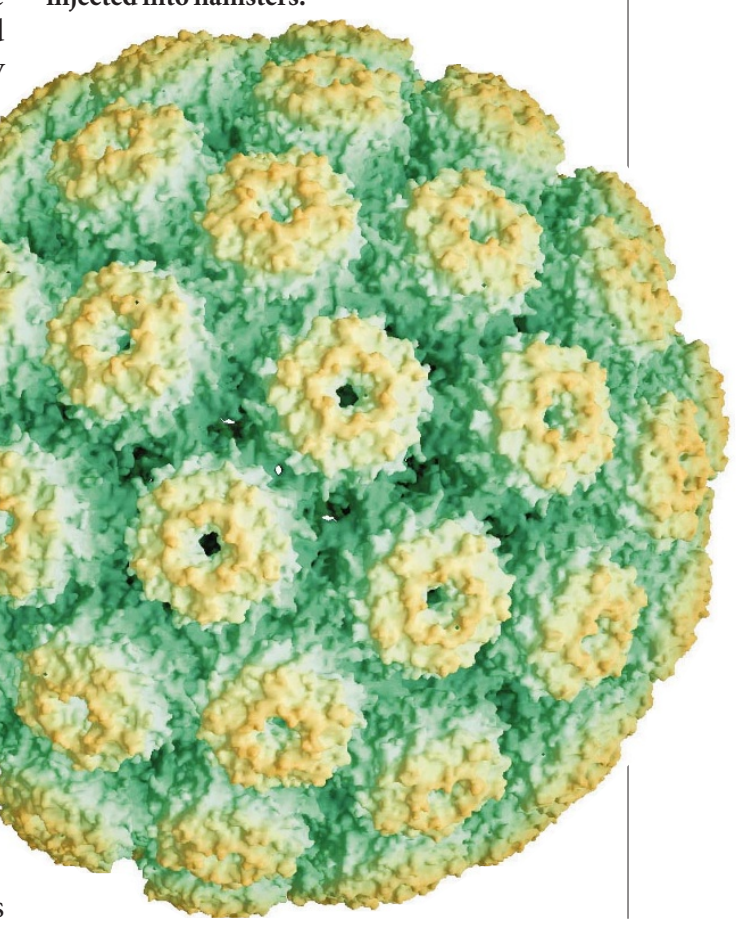

